\title{
Modal Analysis on the Wind Tunnel Test Bed based on Workbench
}

\author{
Zi-yan Shao, Wen-jia Chen ${ }^{a}$, Guan-jian Li, Yong-jin Hu \\ College of Mechanical Engineering, Yangzhou University, Yangzhou, Jiangsu, China
}

\begin{abstract}
This paper shows a wind tunnel test bed with parallelogram structure. The three-dimensional model was built by Solidworks after analyzing the movement principle. Then the model was imported to Workbench and carried out modal analysis according to the theory of finite element modal analysis. Finally the first six vibration mode and the natural frequency were got. This result provides theoretical support for the further research of the stability of the structure, and it also provides theoretical basis for the optimal design of the institution.
\end{abstract}

\section{Introduction}

With the development of science and technology, the development of aircraft has been on the fast track. The wind tunnel test becomes a crucial part from the process of designing to that of being a product. Putting the aircraft model which is reduced according to a certain proportion and then put into the wind tunnel and carrying out the experiment can effectively reduce the time of development effectively. And the key procedure of the experiment is the wind tunnel test bed. The more stable the supporting structure is, the more accurate the test result is $[1,2,3]$.

Taking a wind tunnel test bed which is based on the parallelogram structure as its research subject, this paper utilizes Solidworks to establish a three-dimensional model and then imports it to the Workbench with the purpose of doing a modal analysis and analyzing the modal change. All these provide theoretical support for further researches.

This paper investigates a kind of wind tunnel test bed based on the parallelogram structure. The institution can lead the test model to realize three-dimensional rotation with a constant mass center. The diagram is shown in Figure1. The movements of the structure are divided into three parts, namely, leeway motion, pitch motion and roll motion.

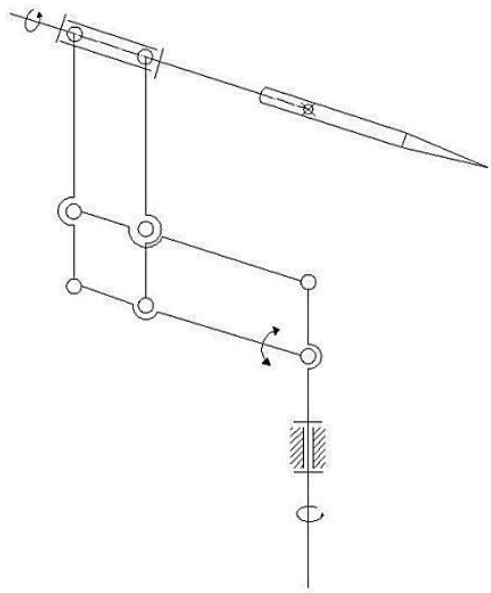

Figure1 The kinematic diagram of the wind tunnel test bed

\section{The Finite Element Modal Analysis Theory}

Modal analysis[4,5] is a kind of modern method of studying the dynamic characteristics of the structure. It is mainly used to determine the vibration characteristics of the structure and the parts of the machine, including natural frequency and vibration. Modal analysis is also the basis of other dynamics analysis, such as harmonic response analysis, transient dynamics analysis, spectrum analysis and so on.

In terms of modal analysis, the vibration frequency and modal are figured out by the following equation:

$$
\left([\mathrm{K}]-\omega_{\mathrm{i}}^{2}[\mathrm{M}]\right)\left\{\varnothing_{\mathrm{i}}\right\}=0
$$

In this equation, the stiffness matrix $[\mathrm{K}]$ and the mass matric $[\mathrm{M}]$ are fixed. And the material needs to be linear elastic and small displacement theory should be used. Also the material doesn't have any damping $[\mathrm{C}]$ and vibration force $[\mathrm{F}]$.

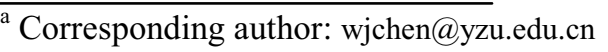


The ultimate goal of the modal analysis is to identify the modal parameters of the system and to provide theoretical foundation for the vibration characters analysis of the system, the diagnosis and forecast of the vibration fault as well as the optimized design of the dynamic characteristics of the structure.

Modal analysis in Workbench is linear. During the process of analysis, the damping effects on its vibration are ignored. Moreover, any force exerted in the modal analysis won't be taken into consideration. Statics analysis needs to be done first before modal analysis. Then the pre-stress in the initial condition of modal analysis needs to be indicated that it comes from the previous static analysis result.

\section{The Establishment of the Finite Element Model}

\subsection{The Built of the 3-D Model}

There is plenty of CAD modeling software, such as SolidWorks, ProE, UG, Catia etc. Solidworks is easy to operate and it also supports parametric design. Therefore, it is chosen as the modeling tool in this paper [6].

The entity model of every separate part is established in SolidWorks and then the assembly model is built according to the cooperative relationship between different parts. In the process of designing, full consideration should be given to the range of movement of each part as well as the whole organization in order to avoid interference and other problems. In order to ensure that the entity structure is identical to the finite element grid and guarantee the modal characteristics of the results in the analysis process, the structure is simplified. At the same time, it is also convenient for computers to conduct analysis. The 3-D model of the wind tunnel test bed building in SolidWorks is shown in Figure2.

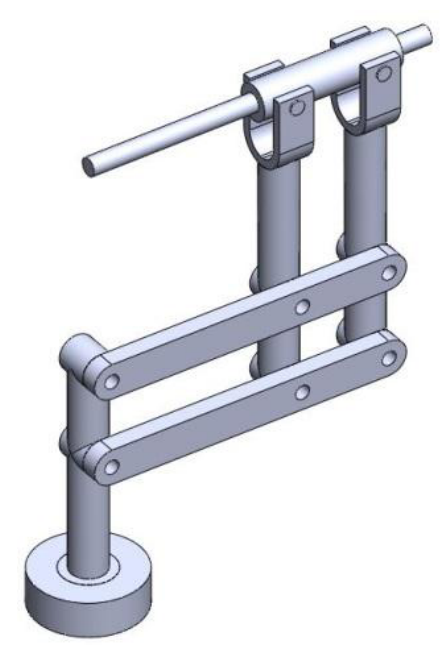

Figure2 The 3-D model of the wind tunnel test bed

In this system, the leeway motion is realized by the driving rod which is fixed on the base. The pitch motion is driven by the parallelogram structure. And the rotating motion is realized by the end rod of the system. The barycentric position of the test model keeps fixed during the whole moving process.

The 3-D entity model which is built is saved as Parasolid, and then imported into ANSYS Workbench to carry out the pretreatment of modal analysis.

\subsection{The Pretreatment of Modal Analysis}

The pretreatment of modal analysis includes setting the properties of the materials, meshing, loading pressure and boundary conditions, as well as setting the frequency option [7,8].

Material properties needing to be used in modal analysis include Young's modulus, Poisson's ratio and density. The priorities of materials in this system are shown in Table1.

Table1 The priorities of materials

\begin{tabular}{cc}
\hline Young's modulus & $71 \mathrm{GPa}$ \\
\hline Poisson's ratio & 0.33 \\
\hline Density & $2.7 \times 103 \mathrm{~kg} / \mathrm{m}^{3}$ \\
\hline
\end{tabular}

Only node and unit of the grid participate in the calculation in the finite element analysis. Therefor the density of the grid can directly affect the accuracy of the result. But the refinement of the grid also increases the time for calculation and the need of more storage space [7]. Meshing platform will generate the default grid when the solution process begins. In the analysis of this institution, based on the method of free division, there are altogether 26937 nodes and 9732 units. The system after dividing the grids is shown in Figure3.

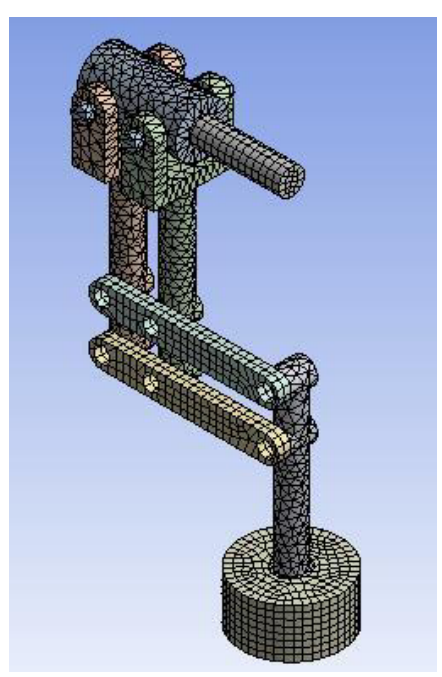

Figure3 The system after dividing the grids

The free modal analysis of the test model supporting institutions doesn't deal with any load or boundary conditions. 


\section{Results of Modal Analysis}

Using modal analysis of Workbench to carry out calculation, we can get the first six stage natural frequency as is shown in Table2

Table2 The first 6 stage natural frequency

\begin{tabular}{cc}
\hline Order & Natural frequency $(\mathbf{H z})$ \\
\hline 1 & 0 \\
\hline 2 & $9.89 \times 10^{-4}$ \\
\hline 3 & $3.46 \times 10^{-3}$ \\
\hline 4 & 14.76 \\
\hline 5 & 17.7 \\
\hline 6 & 45.2 \\
\hline
\end{tabular}

After getting the natural frequency of the institutions, the first six variation models are chosen, which corresponds to their own natural frequency. The first six modal vibration modes are shown in Figure4.

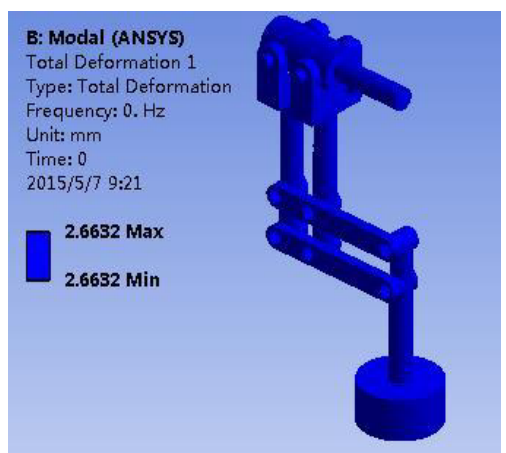

(1) first-stage mode of vibration

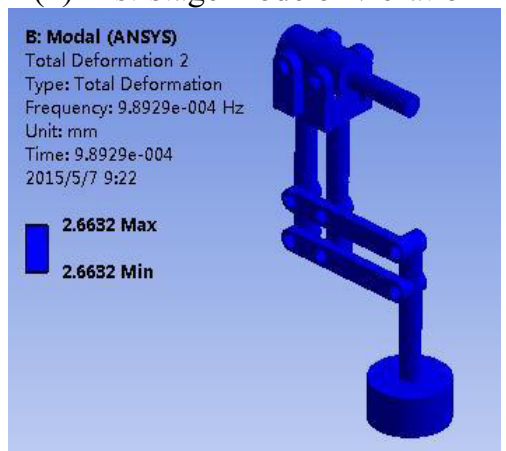

(2) second-stage mode of vibration

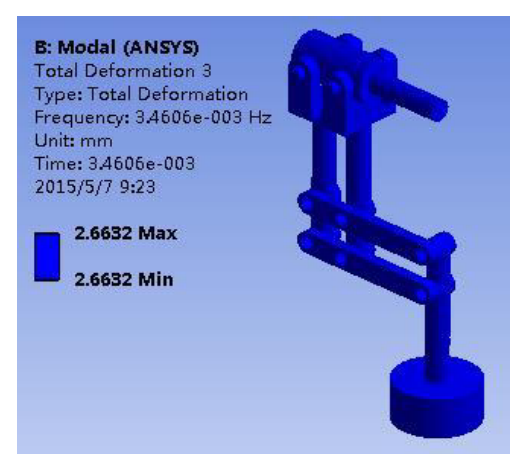

(3) third-stage mode of vibration

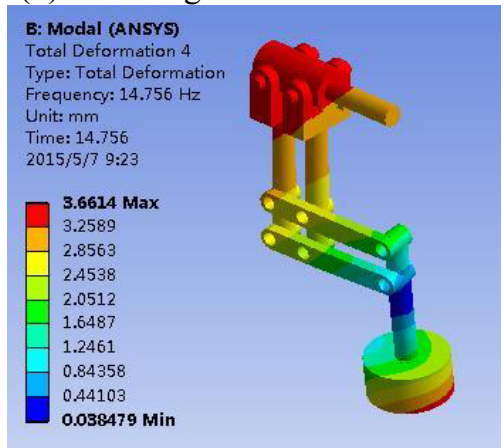

(4)forth-stage mode of vibration

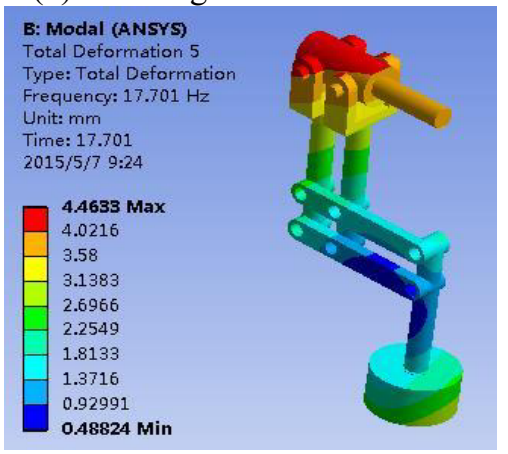

(5) fifth-stage mode of vibration

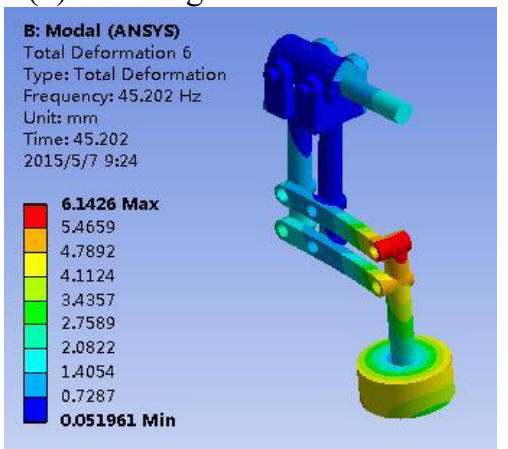

(6) sixth-stage mode of vibration

Figure4 First six modal vibration modes

It can be seen from the results of analysis that the natural frequency of the institution ranges from 0 to $45.20 \mathrm{~Hz}$. Besides the vibration amplitude increases, as the frequency goes up.

\section{Conclusions}

1) After an analysis of the dynamic theory of the wind tunnel test model, the three-dimensional model is built by using SolidWorks. 
2) While the natural frequency of the wind tunnel test model supporting system increases from $0 \mathrm{HZ}$ to $45.20 \mathrm{HZ}$, its vibration exerts an increasing influence on the model.

3) After carrying out modal analysis with the aid of Workbench, it is concluded that the natural frequency has

\section{Acknowledgements}

The authors would like to acknowledge the financial support of the Nature Science Foundation of Jiangsu Province of China (No.BK2012687) and the National Natural Science Foundation of China (No.50975249).

\section{References}

1 Lanlei Zhao. modeling and simulation research of a wind tunnel test support platform with five degree of freedom. (2013)

2 Rein M, et al. AIAA Journal of Aircraft, 45(1 286-291.

3 Bergmann A. Nnfm, 102 59-77 (2009) a great effect on the stability of the institution. Therefore, frequency in this range should be avoided in practical application, and this can help to avoid the impact of resonance.

4 Jian zhang, Wen-jia chen, Lan-lei zhao. Mechanical Engineer, 12 59-62 (2011)

5 Guilong Ling, Jinbin Ding, Zheng Wen (eds) ANSYS Workbench 13.0 from the introduction to the master, Tsinghua University Press: Beijing, 114-136 (2012)

6 Duan Lv, Dongjian Zeng, Xiaoyang Yu, Longping Zhang. Mechinery Design \& Manufacture, 8 11-13 (2012)

7 Bing Li, Zhengjia He, Xuefeng Chen (eds), Design, Simulation and Optimization of ANSYS Workbench, Tsinghua University Press: Beijing (2011)

8 Wenjian Liu (ed) The structural analysis of scanner based on ANSYS Workbench, Xian, Xidian University. (2009) 